\title{
MEASUREMENTS OF THE SPS TRANSVERSE IMPEDANCE IN 2000
}

\author{
G. Arduini, H. Burkhardt, K. Cornelis, J. Klem, F. Zimmermann, M.-P. Zorzano, CERN
}

\section{Abstract}

We report on measurements of coherent tune shifts, head-tail growth rates, and current-dependent betatron phase advances at the CERN SPS in the year 2000. Comparing results obtained at two different energies shows that there is no notable contribution from space charge. Within the measurement resolution the impedance is the same as in 1999, consistent with the expected small effect from changes to only a small number of pumping ports. In 2000, data were taken over an expanded range of chromaticities, which increases the sensitivity to the impedance frequency distribution. Measurements of the current-dependent phase advance around the ring help localizing the most important impedance sources.

\section{INTRODUCTION}

As part of the SPS upgrade as LHC injector the transverse impedance is being reduced by shielding of more than 1000 vacuum pumping ports, which represented about two thirds of the total impedance [1], and by removing lepton components. About $20 \%$ of the pumping ports were already shielded during the winter shut down 1999/2000.

To monitor the effect of the ongoing vacuum-chamber modifications, a measurement program for the SPS transverse impedance was launched in 1999 [2]. The 2000 measurements aimed to (1) reproduce the 1999 data, (2) possibly discern a first small improvement, (3) further develop machine set up and measurement procedure, (4) quantify contributions from space charge, and (5) explore complementary measurement approaches.

All our studies were performed with a single proton bunch. Relevant SPS parameters are listed in Table 1.

Table 1: Relevant SPS parameters.

\begin{tabular}{lcc}
\hline variable & symbol & value \\
\hline momentum & $p$ & $26 \mathrm{GeV} / \mathrm{c}$ \\
circumference & $C$ & $6916 \mathrm{~m}$ \\
betatron tune & $Q_{x, y}$ & $\sim 26.6$ \\
momentum compaction & $\alpha_{C}$ & $1.856 \times 10^{-3}$ \\
bunch population & $N_{b}$ & $1-10 \times 10^{10}$ \\
rms bunch length & $\sigma_{z}$ & $150 \mathrm{~mm}$ \\
rms geom. tr. emittance & $\epsilon_{x, y}$ & $\sim 0.25 \mu \mathrm{m}$ \\
ave. beta function & $\beta$ & $\sim 40 \mathrm{~m}$ \\
\hline
\end{tabular}

The impedance is described by a broadband resonator

$$
Z_{\perp}(\omega)=\frac{\omega_{r}}{\omega} \frac{R_{\perp}}{1+i Q\left(\frac{\omega_{r}}{\omega}-\frac{\omega}{\omega_{r}}\right)},
$$

with resonant frequency $\omega_{r} / 2 \pi=1.3 \mathrm{GHz}$ and quality factor $Q=1$ [3]. Its effect is a complex tune shift [4]

$$
\Delta \omega_{\beta}=-\frac{N_{b} e c}{4 \sqrt{\pi} \omega_{\beta}(p / e) T_{0} \sigma_{z}} i\left(Z_{\perp}\right)_{\mathrm{eff}}
$$

where $T_{0}$ is the revolution frequency, $\omega_{\beta}$ the angular betatron frequency (including the integer part of the tune), and $\left(Z_{\perp}\right)_{\text {eff }}$ the effective transverse impedance. The latter is obtained by convoluting the impedance of Eq. (1) with a Gaussian bunch spectrum $h_{0}(\omega)=\exp \left(-\omega^{2} \sigma_{t}^{2}\right)$ that is shifted in frequency, $\omega \rightarrow \omega-\omega_{\xi}$, by $\omega_{\xi}=\xi \omega_{\beta} / \eta$, where $\xi=Q^{\prime} / Q=\frac{\Delta Q}{Q} / \frac{\Delta p}{p}$ denotes the chromaticity, and $\eta$ the slippage factor. In Eq. (2), we have assumed a constant beta function $\beta=c / \omega_{\beta}$.

We estimate the imaginary part of $Z_{\perp}$ by measuring the coherent tune shift with current, and using $\left(\sigma_{z} \Delta Q_{x, y} / \Delta N_{b}\right) \propto \operatorname{Im} Z_{\text {eff }} \sim R_{\perp} / Q$. The real part is obtained from the growth (or damping) rate $1 / \tau$ of the $l=0$ head-tail mode, measured as a function of chromaticity $\xi$, via $\left(-\sigma_{z} /\left(\tau N_{b}\right)\right) \propto \operatorname{Re}\left(Z_{\perp}\right)_{\text {eff }} \sim \omega_{\xi} / \omega_{r} R_{\perp} / Q^{2}$. The ' $\sim$ ' signs indicate a proportionality in the limit of long bunches $\left(\omega_{r} \sigma_{z} \gg c\right)$. Note that the relation between impedance and coherent tune shift given above strictly applies only for the case of cylindrical symmetry [5]. In the case of asymmetric structures, as in the SPS, part of the coherent tune shift arises from 'detuning' or 'incoherent' wakes, which are excited even if the beam is centered in the chamber. To mark this difference, we add a superindex ' $\Delta Q$ ' to impedance numbers computed from the tune shift.

\section{BEAM PARAMETERS}

Impedance measurements are ideally done at identical conditions, varying only the intensity in case of the coherent tune shift measurements or chromaticity in case of the growth/decay rate measurements. The $200-\mathrm{MHz}$ rf-voltage for the measurements was fixed to a relatively high value, so as to obtain shorter bunches and to enhance the measurable effect. The rf voltage during injection is lower (typically 1.7 MV), and adapted for optimal matching. Between injection/capture and the measurements the rf-voltage is ramped adiabatically over a time span of about $80 \mathrm{~ms}$. Figure 1 illustrates the voltage ramping and bunch-length reduction after injection.

At the moment of the measurements within the SPS cycle, the 200-MHz rf voltage was about 2.6 MV for the studies at $26 \mathrm{GeV} / \mathrm{c}$ and $4.3 \mathrm{MV}$ at $120 \mathrm{GeV}$. In order to establish stable beam parameters and to obtain reproducible results, we systematically monitor the bunch lengths and the transverse emittances. A good knowledge of the bunch length is also required for converting the measured tune shifts or growth rates into an impedance value; see Eq. (2). Figure 2 summarizes bunch length measurements in 2000. 


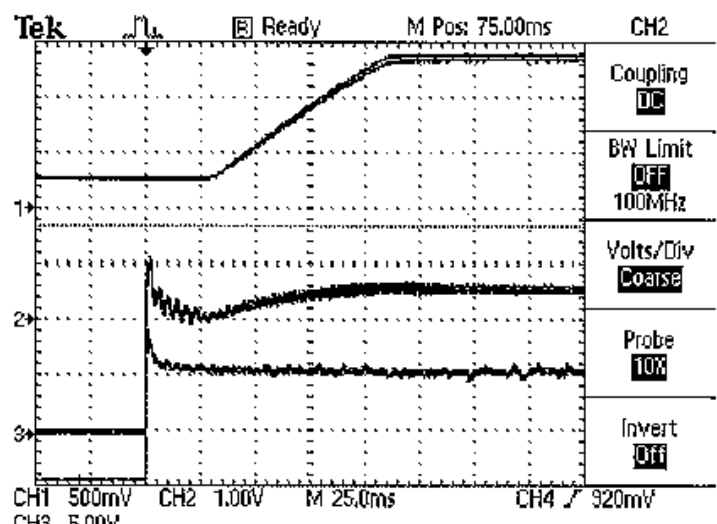

Figure 1: Scope signals illustrating the rf ramp and bunch shortening prior to the measurement. Top trace: rf voltage; medium trace: peak current signal (inversely proportional to the bunch length); bottom trace: dc current signal. The initial decay of both peak and dc currents indicates some beam loss immediately after injection.

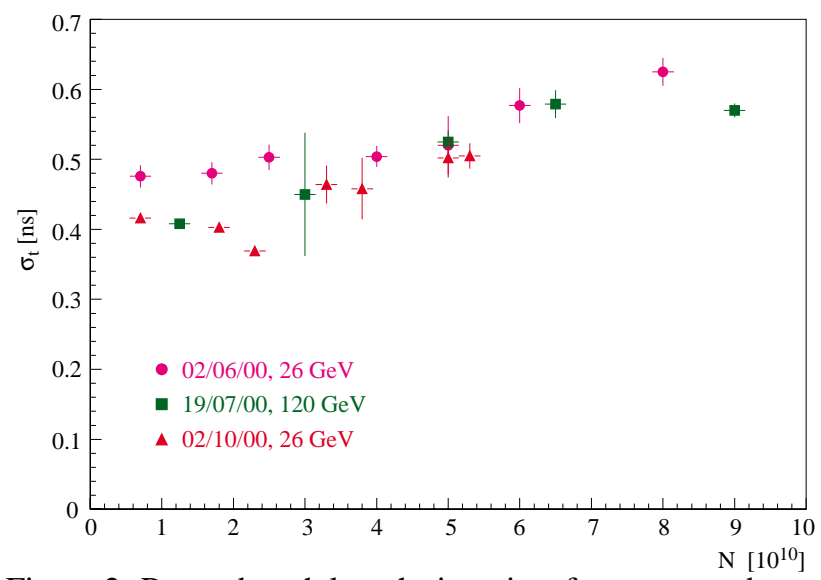

Figure 2: R.m.s. bunch lengths in units of ns measured as a function of bunch population in $10^{10}$ for the three study periods in the year 2000. The values correspond to the standard deviation of a Gaussian that was fitted to the signal from a wide-band pick up, recorded on a scope. The vertical error bars reflect the r.m.s. spread of several measurements taken at the same intensity. The systematic error in the Gaussian fit for a single measurement is about 0.003 ns (not shown). The horizontal error bar approximates the cycle-to-cycle intensity fluctuation.

The bunch intensities were varied between 1 and $10 \times$ $10^{10}$ protons by vertical scraping in the SPS. We used the rotational wire scanner to monitor the emittances. The horizontal emittance was rather constant. The vertical emittance varied by more than a factor of 10 , due to the scraping. To minimize the bias of our measurement from this effect, we empirically adjusted the octupole magnets so as to cancel the detuning with amplitude.

\section{TUNE SHIFT AND GROWTH RATE}

Tune spectra are obtained by applying a fast Fourier transform (FFT) to samples of $2^{12}=4096$ successive turns. Precise tune values are calculated off-line using an optimized algorithm with windowing and peakinterpolation. A result for $120 \mathrm{GeV}$ is shown in Fig. 3. The scattering of individual measurements implies an uncertainty of $4 \times 10^{-5}$ on individual tune measurements.

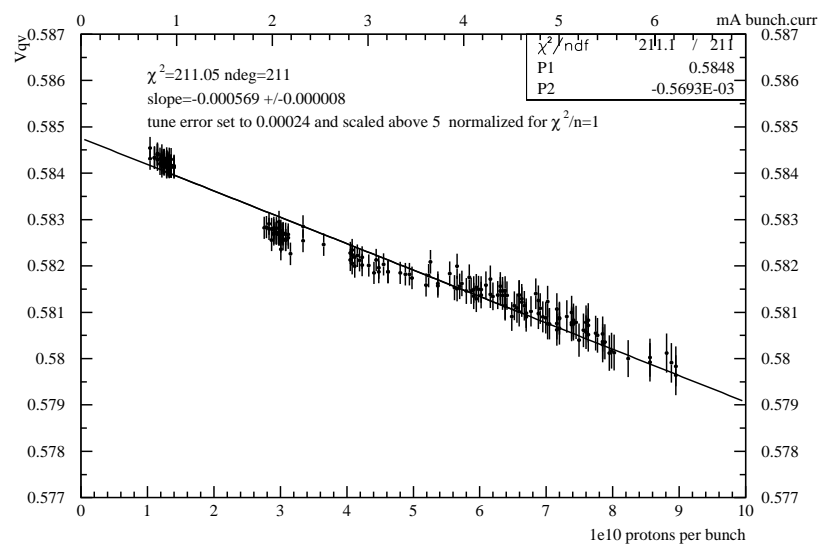

Figure 3: Vertical coherent tune shift versus bunch population at $120 \mathrm{GeV}(19 / 07 / 00)$.

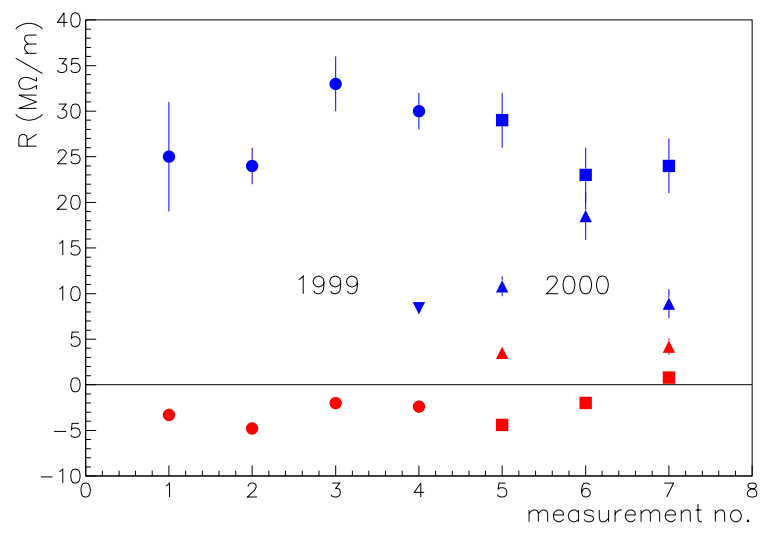

Figure 4: Impedance values $R_{\perp}^{(\Delta Q)}, x(y)$ inferred from all measurements in 1999 (first 4) and 2000 (the last 3), by fitting to a broadband resonator with resonant frequency $\omega_{r} / 2 \pi=1.3 \mathrm{GHz}$ and quality factor $Q=1$ [3]. Triangles are the $R_{\perp}$ values obtained from the growth rate measurements; squares and circles represent $R_{\perp}^{\Delta Q}$ inferred from the coherent tune shift. The 6 th measurement was performed at $120 \mathrm{GeV}$, all others at $26 \mathrm{GeV}$.

Together with the measured bunch length and the knowledge of the beam energy, the effective transverse impedance can be computed from Eq. (2). The results are shown in Fig. 4 (the circles and squares), which suggests that $\operatorname{Im}\left(Z_{\perp}\right)_{\text {eff }}$ was measured reproducibly on the 10-20\% level. The average vertical values in 1999, $R_{\perp, y}^{\Delta Q}=28 \pm 2$ $\mathrm{M} \Omega / \mathrm{m}$, and in $2000, R_{\perp, y}^{\Delta Q}=26 \pm 3 \mathrm{M} \Omega / \mathrm{m}$, are compatible with each other, and might indicate a small impedance decrease by about $10 \%$. The corresponding values in the horizontal plane are $R_{\perp, x}^{\Delta Q}=-2.6 \pm 0.2 \mathrm{M} \Omega / \mathrm{m}$ for 1999 , and $R_{\perp, x}^{\Delta Q}=-0.9 \pm 1.8 \mathrm{M} \Omega / \mathrm{m}$ for 2000 . The horizon- 
tal tune shift is almost zero. This is the expected result for a flat chamber, where effects of coherent and incoherent wakes cancel each other [5]. If scaled by the momentum, the $120-\mathrm{GeV}$ impedance measurement (no. 6 in Fig. 4), is consistent with the $26-\mathrm{GeV}$ value. Hence, the space-charge contribution at $26 \mathrm{GeV}$ appears to be small.

Growth rates were measured with the same set up, but by varying the chromaticity instead of intensity. Figure 5 shows a result at $120 \mathrm{GeV}$. The data are compatible with a straight line, as expected for $\left|\omega_{\xi}\right| \ll \omega_{r}$. The top scale in Fig. 5 is directly in units of frequency. By fitting the real part of the effective impedance, the measured slope can be translated into a value of $R_{\perp}$, via Eq. (2). Results of the growth rate measurements are also depicted in Fig. 4 (the triangles). The corresponding average impedances are $R_{\perp, y}=11.1 \pm 2.7 \mathrm{M} \Omega / \mathrm{m}$, and $R_{\perp, x}=3.7 \pm 0.3 \mathrm{M} \Omega / \mathrm{m}$.

The incoherent wake fields in the vertical and horizontal planes are of equal magnitude, but of opposite sign [5]. Assuming $\beta_{x} \approx \beta_{y}$ at the wake-field locations, the following relation should hold: $R_{\perp, y}=\left[R_{\perp, y}^{\Delta Q}-\left(R_{\perp, x}-R_{\perp, x}^{\Delta Q}\right)\right]$. The two sides of this equation evaluate to 11 and $20 \mathrm{M} \Omega / \mathrm{m}$, respectively, which suggests that the actual resonator $Q$ value is about 2 , rather than 1 .

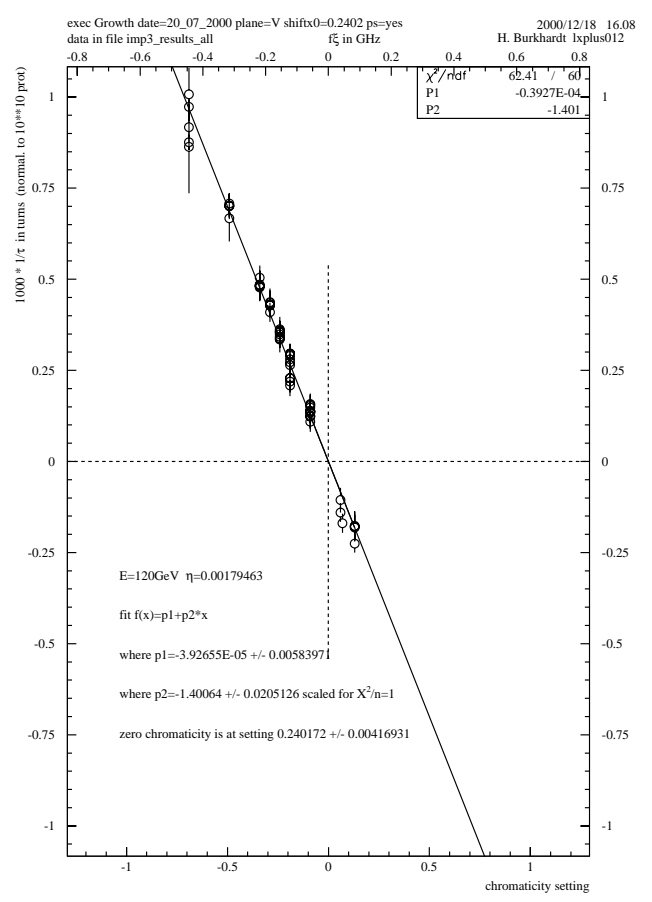

Figure 5: Growth rate of vertical head-tail mode (in units of $10^{-3}$ turns $^{-1}$ ) measured as function of chromaticity $\xi$ at $120 \mathrm{GeV}$ on $19 / 07 / 2000$.

\section{IMPEDANCE LOCALIZATION}

The SPS is equipped with about 100 position monitors per plane. These can be read out in multi-turn mode, thereby providing the possibility for localized impedance measurements as pioneered in LEP [6, 7]. From multi-turn data at all BPMs, the accumulated phase advance around the ring can be computed [8]. Repeating this measurement for two different beam currents and subtracting the two results then yields the change in the phase advance with beam current. An example is shown in Fig. 6. With present errors it is hard to draw a definite conclusion. But there could be a first indication of steps (non-negligible local impedance contributions) in the injection, rf and extraction area (located roughly at 1000, 3000 and $6000 \mathrm{~m}$ ).

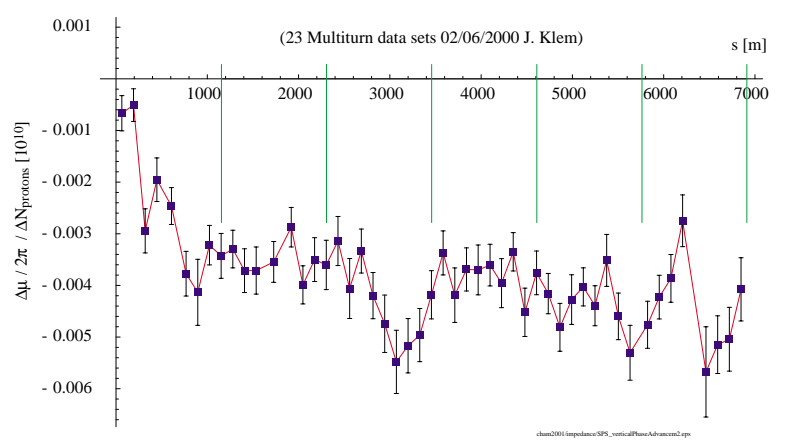

Figure 6: Change of the accumulated vertical phase advance around the SPS ring with bunch intensity [7].

\section{OUTLOOK}

In 2001 we should be able to quantify the impedance reduction achieved by the SPS upgrade programme. In addition, we will try to further extend the range of chromaticities in the growth-rate measurements, so as to enhance the sensitivity to resonant frequencies of individual impedance components. According to a preliminary measurement in 2001, the signal of the head-tail monitor [9] strongly varies with beam current. This could be exploited for a direct observation of the impedance effect on the $l=1$ head-tail mode. Together with the coherent tune shift of the $l=0$ mode, this might allow us to distinguish experimentally the coherent and incoherent wake contributions.

Further informations can be found in Refs. [10].

\section{ACKNOWLEDGEMENTS}

We thank P. Baudrenghien, L. Normann, M. Albert, J. Gareyte, D. Brandt, L. Vos, G. Rumolo, R. Jones, and the PS division for help and discussions.

\section{REFERENCES}

[1] L. Vos, CERN SPS/86-21 (MS).

[2] G. Arduini, et al., EPAC2000 Vienna (2000).

[3] D. Boussard, J. Gareyte, Improvement Report no. 181 (1980).

[4] F. Sacherer, Proc. 1976 Erice, CERN 77-13m, p. 198 (1977).

[5] J. Gareyte, CERN SL/AP forum, 3. April 2001; K. Yokoya, Part. Acc. 41, p. 221 (1993); V. Danilov, VLHC workshop, SLAC, March 2001.

[6] D. Brandt, et al., IEEE PAC95 Dallas (1995).

[7] J. Klem, et al., Proc. EPAC 2000 Vienna (2000).

[8] P. Castro et al., IEEE PAC 93, Washington, p. 2103 (1993).

[9] D. Cocq et al., 8th WBI, Stanford, p. 281 (1998).

[10] G. Arduini et al., SL-Note-2001-002 MD; and H. Burkhardt, Chamonix XI, CERN-SL-2001-003 DI (2001). 